\title{
KIBRA Polymorphism Is Associated with Individual Differences in Hippocampal Subregions: Evidence from Anatomical Segmentation using High-Resolution MRI
}

\author{
Daniela J. Palombo, ${ }^{1,2}$ Robert S.C. Amaral, ${ }^{1}$ Rosanna K. Olsen, ${ }^{1,2}$ Daniel J. Müller, ${ }^{3,4}$ Rebecca M. Todd, ${ }^{5}$ \\ Adam K. Anderson, ${ }^{6}$ and Brian Levine ${ }^{1,2,7}$ \\ ${ }^{1}$ Rotman Research Institute at Baycrest Hospital, Toronto, Ontario M6A 2E1 Canada, ${ }^{2}$ Department of Psychology, University of Toronto, Toronto, Ontario \\ M5R 0A3 Canada, ${ }^{3}$ Centre for Addiction and Mental Health, Toronto, Ontario M5T 1R8 Canada, ${ }^{4}$ Department of Psychiatry, University of Toronto, Toronto, \\ Ontario M6A 2E1 Canada, ${ }^{5}$ Department of Psychology, University of British Columbia, Vancouver, British Columbia V6T 1Z4 Canada, ${ }^{6}$ Department of \\ Human Development, Cornell University, Ithaca, New York 14853, and 7Department of Medicine (Neurology), University of Toronto, Toronto, Ontario \\ M6A 2E1 Canada
}

The KIBRA gene has been associated with episodic memory in several recent reports; carriers of the T-allele show enhanced episodic memory performance relative to noncarriers. Gene expression studies in human and rodent species show high levels of KIBRA in the hippocampus, particularly in the subfields. The goal of the present study was to determine whether the KIBRA C $\rightarrow \mathrm{T}$ polymorphism is also associated with volume differences in the human hippocampus and whether specific subfields are differentially affected by $K I B R A$ genotype. High-resolution magnetic resonance imaging (T2-weighted, voxel size $=0.4 \times 0.4 \mathrm{~mm}$, in-plane) was used to manually segment hippocampal cornu ammonis (CA) subfields, dentate gyrus (DG), and the subiculum as well as adjacent medial temporal lobe cortices in healthy carriers and noncarriers of the KIBRA T-allele (rs17070145). Overall, we found that T-carriers had a larger hippocampal volume relative to noncarriers. The structural differences observed were specific to the CA fields and DG regions of the hippocampus, suggesting a potential neural mechanism for the effects of KIBRA on episodic memory performance reported previously.

\section{Introduction}

Several recent studies have identified genetic variations and molecular pathways that are associated with episodic memory performance (for review, see Papassotiropoulos and de Quervain, 2011), which is the type of memory that enables recollection of specific events (Tulving, 2002). In the first genome-wide association study of human memory, an association between a single nucleotide polymorphism (SNP) of the kidney and brain $(K I B R A)$ gene $(\mathrm{C} \rightarrow \mathrm{T})$ and episodic memory was first identified (Papassotiropoulos et al., 2006). In three cohorts, T-allele carriers (TT/TC) of the rs 17070145 SNP showed an advantage in episodic memory performance relative to noncarriers (CC). This finding

Received April 1, 2013; revised June 25, 2013; accepted July 9, 2013.

Author contributions:D.J.P., R.K.O., D.J.M., R.M.T., A.K.A., and B.L. designed research;D.J.P., R.S.C.A., and R.K.O. performed research; D.J.P. analyzed data; D.J.P. wrote the paper.

This research was supported by a grant from the Canadian Institutes of Health Research (CIHR) to B.L. (MOP62963) and A.K.A. (MOP-491746). D.J.M. is supported by CIHR Michael Smith New Investigator Salary Prize, Ontario Mental Health Foundation New Investigator Fellowship, and an Early Researcher Award by the Ministry of Research and Innovation of Ontario. D.J.P. was supported by the Ontario Graduate Scholarship. We thank Dr. Malcolm Binns for statistical consulting and Dr. Valerie Carr for advice about the segmentation protocol. We also thank Amanda Robertson and Natalie Freeman for recruitment assistance and genotyping, respectively. Finally, we acknowledge Nick Qiu and Drs. Melissa Pangelinan, Erin Dickie, and M. Mallar Chakravarty for assistance with the T1 pipeline.

The authors declare no competing financial interests.

Correspondence should be addressed to Brian Levine, Rotman Research Institute at Baycrest, 3560 Bathurst Street, Toronto, 0N M6A 2E1, Canada. E-mail: blevine@research.baycrest.org.

DOI:10.1523/JNEUROSCI.1406-13.2013

Copyright $\odot 2013$ the authors $\quad 0270-6474 / 13 / 3313088-06 \$ 15.00 / 0$ has been replicated several times, although there have been null findings (for a meta-analysis, see Milnik et al., 2012).

Given the well documented role of the hippocampus in episodic memory (Eichenbaum et al., 2007), the goal of the present study was to determine whether the KIBRA C $\rightarrow$ T polymorphism is also associated with volumetric differences in the human hippocampus. KIBRA expression is high in the hippocampus in both humans and rodents (Papassotiropoulos et al., 2006; Johannsen et al., 2008; Yoshihama et al., 2009). Moreover, KIBRAdependent differences in blood oxygenation level-dependent (BOLD) response in the hippocampus and medial temporal lobe (MTL) cortices have been observed during episodic remembering (Papassotiropoulos et al., 2006; Kauppi et al., 2011), although these studies actually yielded opposite results (i.e., increased activation in noncarriers vs increased activation in T-carriers, respectively). Yet, null genotype differences in whole hippocampal and MTL cortex volume have been observed in one report that used both manual and automated magnetic resonance imaging (MRI) segmentation (Papassotiropoulos et al., 2006). However, the hippocampus consists of histologically heterogeneous subregions (i.e., cornu ammonis (CA) fields 1-3, dentate gyrus (DG), subiculum) with distinct patterns of connectivity and cellular structure (Amaral and Insausti, 1990; Duvernoy, 2005). Papassotiropoulos et al. (2006) demonstrated that KIBRA is most highly expressed in the $\mathrm{CA}_{1}$ and DG in rodents; likewise Johannsen (2008) observed intense KIBRA expression in DG granule cells and CA pyramidal cells in rodents (also see Yoshihama et 
al., 2009), raising the possibility that whole-hippocampal volume may be a less sensitive marker to assess KIBRA-dependent structural variability in humans. To assess regional differences in the effects of KIBRA genotype on hippocampal structure, we conducted MRI-based volumetric analyses of hippocampal and adjacent MTL cortical subregions. We hypothesized that KIBRAdependent differences in hippocampal volume would be localized within the hippocampal CA fields and DG, paralleling the expression patterns observed in rodents.

\section{Materials and Methods}

Participants. Thirty-two healthy Caucasian individuals, stratified by KIBRA genotype (T-carriers and noncarriers), were recruited from a larger study investigating genetics and memory. Genotype groups were matched on genetic characteristics previously implicated in episodic memory: apolipoprotein E (APOE) $\varepsilon 4$ alleles, brain-derived neurotropic factor (BDNF) Val66Met, and catechol-O-methyltransferase (COMT) Val158Met. To complement methodology used by Papassotiropoulos et al. (2006), participants were also one-to-one matched (within $0.4 \mathrm{SDs}$ ) between genotype groups on old/new visual recognition memory performance as assessed within 2 years of scanning (for task details, see Rudebeck et al., 2009). Due to equipment failure, imaging data were unavailable for four noncarriers. Thus our sample included 18 T-carriers ( $22.2 \pm 3.7$ years old; $15.5 \pm 2.8$ years of education; 4 male) and 14 noncarriers $(20.3 \pm 3.0$ years old; $13.9 \pm 1.6$ years of education; 3 male). This did not substantively affect the balance between genotype groups on the aforementioned variables: APOE $\varepsilon 4$ alleles $(p=0.57)$; BDNF Val66Met ( $p=0.77)$; or COMT Val158Met ( $p=0.96 ; \chi^{2}$ tests). Genotype groups also did not significantly differ in visual recognition memory scores $\left(p=0.78, \mathrm{hp}^{2}=0.003\right)$. While it is acknowledged that performance differences attributable to genotype are unlikely to be detected in this sample size, our results may nonetheless help to explain findings from other larger studies where performance effects are observed.

Given previous research that sex modifies the relationship between KIBRA and cognition (Wersching et al., 2011), sex was included as a covariate in all analyses, although this did not change the pattern of results. There was no significant difference in age between groups $(p=$ $\left.0.13, \mathrm{hp}^{2}=0.08\right)$. There was a marginal group difference in education $\left(F_{(1,29)}=3.65, p=0.07, \mathrm{hp}^{2}=0.11\right)$. As education was not found to be associated with the volume of any regions of interest (ROIs) within genotype group, it was not included as a covariate. This study was approved by local ethics. Participants provided written informed consent and were compensated $\$ 50$ for the MRI.

Genotyping. Participants provided a saliva sample $(\sim 2 \mathrm{ml})$ in an Oragene OG-500 DNA kit (DNA Genotek). Five SNPs across four genes were genotyped using a TaqMan predesigned assay: COMT Val158Met (rs4680); BDNF Val66Met (rs6265); KIBRA (rs17070145); and both the ApoE 112 (rs429358) and 158 polymorphism (rs7412; Life Technologies). For each reaction, $20 \mathrm{ng}$ of genomic DNA was amplified and scaled to a total volume of $10 \mu \mathrm{l}$ in an Applied Biosystems (AB) 2720 thermal cycler. Post amplification products were analyzed on the ABI Prism 7500 Sequence Detection System using the allelic discrimination option and genotype calls were determined manually by comparison to six No Template Controls. For the ApoE markers, the 112 and 158 genotypes were combined to determine participants' ApoE $\varepsilon$ diplotype. Genotyping of $10 \%$ of the samples was replicated for quality control with no discrepancies. Following previous studies (Milnik et al., 2012) we combined all individuals carrying the T-allele (i.e., TT/TC vs CC).

MRI acquisition. Structural images were acquired using a 3 T Siemens Trio scanner. For segmentation, high-resolution T2-weighted images were acquired in an oblique-coronal plane; slices were arranged perpendicular to the long axis of the hippocampus (TE/TR $=68 \mathrm{~ms} / 3000,2-28$ oblique-coronal slices depending on head size, $512 \times 512$ acquisition matrix, voxel size $=0.43 \times 0.43 \times 3 \mathrm{~mm}$, no skip, FOV $=220 \mathrm{~mm}$ ). The first slice was placed slightly anterior of the collateral sulcus; the last slice was placed just before the full superior-inferior extent of the ventricles. To confirm the placement of slices according to these boundaries, a
Table 1. Dice reliability values for intrarater (intra) and inter-rater reliability (inter)

\begin{tabular}{llllll}
\hline & \multicolumn{2}{l}{ Intra } & & \multicolumn{2}{l}{ Inter } \\
\cline { 2 - 3 } \cline { 6 - 6 } & $\mathrm{L}$ & $\mathrm{R}$ & & $\mathrm{L}$ & $\mathrm{R}$ \\
\hline $\mathrm{CA}_{1}$ & 0.85 & 0.86 & & 0.76 & 0.70 \\
$\mathrm{DG} / \mathrm{CA}_{2 / 3}$ & 0.89 & 0.90 & & 0.84 & 0.81 \\
Sub & 0.80 & 0.77 & & 0.70 & 0.67 \\
PRC & 0.84 & 0.88 & & 0.73 & 0.77 \\
ERC & 0.83 & 0.85 & & 0.69 & 0.72 \\
PHC & 0.92 & 0.91 & 0.78 & 0.83 \\
\hline
\end{tabular}

$L$, left; $R$, right.

whole-brain anatomical MRI was first acquired, using a 3D T1-weighted MP-RAGE $(\mathrm{TE} / \mathrm{TR}=2.63 \mathrm{~ms} / 2000 \mathrm{~ms}, 176$ oblique-axial slices, $256 \times$ 192 acquisition matrix, voxel size $=1 \mathrm{~mm}^{3}$, FOV $=256 \mathrm{~mm}$ ). The T1-weighted images were also used to obtain a measure of total brain volume (TBV; see below).

Segmentation. ROI segmentation was completed in participants' native space (coronal plane; Fig. 1). Akin to most previous studies (Mueller et al., 2007; Olsen et al., 2009, 2013; Chen et al., 2011) subregions were not segmented across the entire long axis of the hippocampus. Instead, segmentation was completed only within the middle section, where the dark bands separating hippocampal layers are clear. This corresponded mainly to the body of the hippocampus; however, we also demarcated the most posterior head slices, where the subfields can also be differentiated (Carr et al., 2010; Chen et al., 2011) (for an alternative approach strictly excluding head slices see Mueller et al., 2007). We segmented $\mathrm{CA}_{1}$, subiculum, and $\mathrm{DG} / \mathrm{CA}_{2 / 3}$ with the latter grouped into a single ROI, as these regions cannot be reliably delineated at this resolution. Combined ROIs (i.e., without subfield demarcation) were used to define the remaining head and tail hippocampal slices, which afforded a measure of full hippocampal volume. Outside the hippocampus, the MTL cortices were segmented: entorhinal cortex (ERC), perirhinal cortex (PRC), and parahippocampal cortex (PHC).

Segmentation was performed in FSLview (v3.1.2) by a single rater, blind to group status (Fig. 1). Segmentation was guided by standard anatomical guidelines (Amaral and Insausti, 1990; Insausti et al., 1998; Duvernoy, 2005; Fig. 1) following the procedures described for MTL subregion segmentation for hi-resolution functional MRI (fMRI) by Olsen et al. (2009); which are similar to those used to assess subregion volumetrics (Mueller et al., 2007; Olsen et al., 2013).

Intrarater and inter-rater reliability were established by comparing segmentations of five participants' brains segmented twice by the same rater (repeated with a 2-6 month interval) and to that of a second rater (Table 1). Reliability was assessed using the Dice overlap metric (Dice, 1945), which was computed for each ROI within each hemisphere (= 2 (intersected region $) /($ region $\mathrm{A}+$ region $\mathrm{B}) ; 0=$ no overlap; $1=$ perfect overlap). Dice values were comparable to those reported previously (Bonnici et al., 2012). TBV estimates were acquired from the T1weighted images using an adapted version of the ANIMAL algorithm (Collins et al., 1995). Genotype groups differed marginally in TBV $\left(F_{(1,29)}=3.00, p=0.09, \mathrm{hp}^{2}=0.09\right)$; noncarriers had a larger TBV relative to T-carriers. TBV was accounted for in each ROI using a regression-based technique; each ROI was regressed on TBV (collapsed across groups); and the residual value (i.e., the structure's actual size minus its predicted value based on the individual's TBV) was accounted for in each ROI for each individual (Arndt et al., 1991). All analyses reported below were performed on TBV-adjusted ROI values.

\section{Results}

Group differences in MTL subregion volumes (TBV adjusted) were assessed with three mixed-design ANCOVAs (i.e., whole unsegmented hippocampus, segmented hippocampus, segmented MTL cortex) and post hoc tests. ANCOVAS included KIBRA genotype as a between-subjects factor and laterality and ROI as within-subjects factors (ROI modeled only for the latter two ANCOVAs). All models included sex as a covariate, as noted 


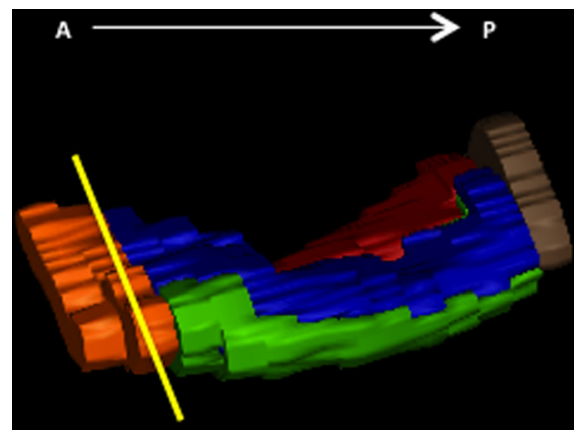

UNSEGMENTED

S
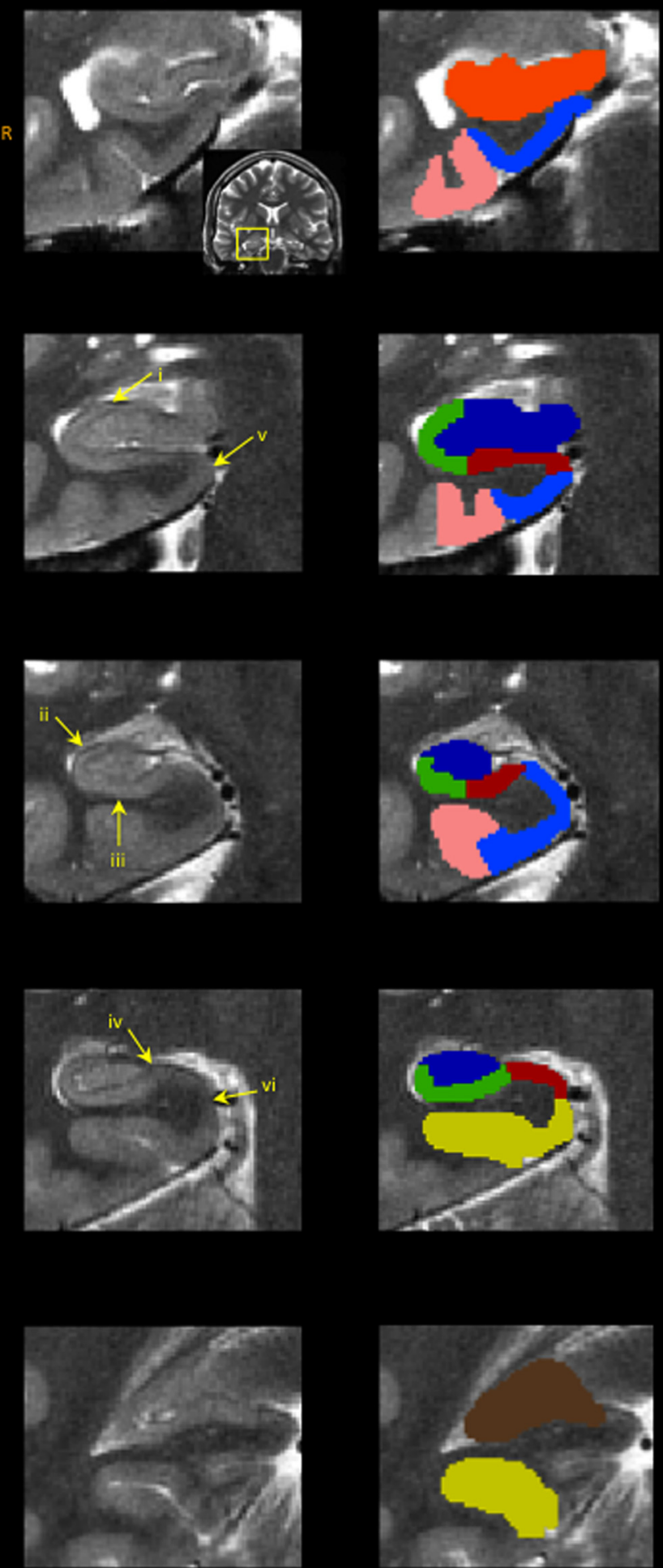

PRC

Figure 1. Slices from T2-weighted $(0.4 \times 0.4 \mathrm{~mm})$ images through the MTLs for one representative participant. Left, Depicts a 3D rendering of the hippocampus. Middle and Right, Show coronal slices through MTL. Subfields were drawn where a clear "C-shape" was discernible, which included all of the body and extended into the most posterior head slices (Zeineh et al., 2000; 0lsen et al., 2009; the remaining head slices and the entire tail included all subfields). Demarcation varied across the long axis (Amaral and Insausti, 1990). Anteriorly, the lateral (superior) boundary of $C_{1} A_{1}$ was drawn by bisecting the most lateral undulation of hippocampus (i). Moving posteriorly, the $\mathrm{CA}_{1}$ was drawn $3 / 4$ of the way up the lateral bend of hippocampus (ii), and its medial extension bisected the $D G / \mathrm{CA}_{2 / 3}$ regions (iiii). In the most posterior slices of the body, $C A_{1}$ was drawn $3 / 4$ of the way up the lateral bend of the hippocampus, and its medial extension was drawn in line with the medial extent of the "tear-drop"-shaped DG/CA $($ iv). Regions extending superior and medial to the $\mathrm{CA}_{1}$ were taken as $\mathrm{DG} / \mathrm{CA}_{2 / 3}$ (Zeineh et al., 2000). Anteriorly, the medial portion of the subiculum (sub) extended until the elbow of the isthmus (v) and in more posterior slices the medial subicular border was drawn halfway down the bend of the isthmus (vi). PRC, ERC, and PHC were segmented according to Insausti et al. (1998). R, right; 5 , superior; A, anterior; P, posterior. 
Table 2. Mean volumes ( $\mathrm{mm}^{3}$; corrected for TBV) are shown for each genotype group for each ROI, hippocampal subregions

\begin{tabular}{|c|c|c|c|c|c|c|}
\hline & \multicolumn{2}{|l|}{$\mathrm{CA}_{1}{ }^{*}$} & \multicolumn{2}{|c|}{$\begin{array}{l}\text { Hippocampal subregions } \\
\text { DG/CA }{ }_{2 / 3}{ }^{*}\end{array}$} & \multicolumn{2}{|l|}{ Sub } \\
\hline & $C C$ & $\mathrm{TT} / \mathrm{TC}$ & $C C$ & $\mathrm{TT} / \mathrm{TC}$ & CC & $\mathrm{TT} / \mathrm{TC}$ \\
\hline L & $594(18)$ & $659(15)$ & 1029 (35) & 1099 (41) & 505 (18) & $476(16)$ \\
\hline $\mathrm{R}$ & $595(29)$ & $692(26)$ & 1079 (37) & $1201(47)$ & 491 (18) & $470(10)$ \\
\hline
\end{tabular}

Volumes are shown for the left $(\mathrm{L})$ and right $(\mathrm{R})$ hemispheres. Standard error of the mean is shown in parentheses. Within the hippocampus, $C A_{1}, D G / C A_{2 / 3}$, and subiculum (sub) were segmented $\left({ }^{*} p<0.05\right)$.

Table 3. Mean volumes ( $\mathrm{mm}^{3}$; corrected for TBV) are shown for each genotype group for each ROI, MTL cortices

\begin{tabular}{|c|c|c|c|c|c|c|}
\hline & \multicolumn{2}{|l|}{ PRC } & \multicolumn{2}{|c|}{$\begin{array}{l}\text { MTL cortices } \\
\text { ERC }\end{array}$} & \multicolumn{2}{|l|}{$\mathrm{PHC}^{+}$} \\
\hline & $C C$ & TT/TC & $C C$ & TT/TC & $C C$ & TT/TC \\
\hline L & 2904 (159) & 3254 (254) & $1100(50)$ & 1074 (32) & 1972 (124) & $2194(80)$ \\
\hline $\mathrm{R}$ & 2611 (163) & 2647 (170) & $1035(51)$ & 1040 (44) & $1898(86)$ & $2102(59)$ \\
\hline
\end{tabular}

Within MTL cortex, ERC, PRC, and PHC were segmented; ${ }^{+} p<0.10$.

above, and a correction for violation of sphericity was applied for repeated measures. Since laterality did not interact with genotype ( $p>0.10$ for all comparisons), it was not interpreted. First, to complement methodology used by Papassotiropoulos et al. (2006), we conducted an ANCOVA on the full hippocampus (i.e., collapsing across all hippocampal ROIs including the head and tail within each hemisphere). Contrary to their findings, we observed larger hippocampal volume in T-carriers relative to noncarriers $\left(F_{(1,29)}=4.70, p=0.04, \mathrm{hp}^{2}=0.14\right)$. Next, we conducted an ANCOVA on the segmented hippocampus, with $\mathrm{ROI}$ as a within-subjects factor (i.e., $\mathrm{CA}_{1}$ and $\mathrm{DG} / \mathrm{CA}_{2 / 3}$, subiculum). This analysis revealed a significant ROI $\times$ Group interaction $\left(F_{(1.97,49.87)}=4.63, p=0.01, \mathrm{hp}^{2}=0.14\right.$, Huynh-Feldt correction), and the main effect of KIBRA genotype was also significant $\left(F_{(1,29)}=5.35, p=0.03, \mathrm{hp}^{2}=0.16\right.$; see Tables 2, 3). Post hoc ANCOVAs for each ROI were performed to explore the nature of this interaction. T-carriers had a larger $\mathrm{CA}_{1}\left(F_{(1,29)}=\right.$ 8.51, $\left.p=0.007, \mathrm{hp}^{2}=0.23\right)$ and DG/CA $2 / 3\left(F_{(1,29)}=4.21, p=\right.$ $\left.0.049, \mathrm{hp}^{2}=0.13\right)$. No group differences were found for subicu$\operatorname{lum}\left(p=0.20 \mathrm{hp}^{2}=0.06\right.$; indeed, noncarriers had a numerically larger volume relative to T-carriers in this region). Finally, we examined the effects of KIBRA genotype on MTL cortex volume, with ROI as a within-subjects factor (i.e., PRC, ERC, PHC), to determine the specificity of the observed effects. This analysis did not reveal group differences $\left(p=0.24, \mathrm{hp}^{2}=0.05\right)$ or interaction $\left(p=0.43, \mathrm{hp}^{2}=0.03\right)$.

\section{Discussion}

We investigated the effects of KIBRA genotype on hippocampal and MTL volume using structural imaging. We provide preliminary novel evidence that the KIBRA T-allele is specifically associated with larger $\mathrm{CA}_{1}$ and $\mathrm{DG} / \mathrm{CA}_{2 / 3}$ volume in young adults, suggesting a putative neural mechanism for the effects of KIBRA genotype on episodic memory reported in the literature (Milnik et al., 2012). In terms of subregion specificity, rodent work suggests functional dissociation within the hippocampus, with $\mathrm{CA}_{1}$ implicated in late retrieval and consolidation processes, while $\mathrm{DG} / \mathrm{CA}_{2 / 3}$ is implicated in encoding and early retrieval (for review, see O'Reilly and Rudy, 2001; Rolls and Kesner, 2006). Similar differentiation has been demonstrated in healthy older adults (Yassa et al., 2011) and clinical populations (Mueller et al., 2011, 2012; Kerchner et al., 2012). However, because these studies ex- amined older adults and patients, it is not known whether they extend to young, healthy individuals.

Interestingly, Papassotiropoulos et al. (2006) observed effects of KIBRA on delayed memory in all three cohorts (ranging from $5 \mathrm{~min}$ to $24 \mathrm{~h}$ ), yet no effects on immediate recall, aligning the effect with the functioning of $\mathrm{CA}_{1}$. A similar pattern was observed by Bates et al. (2009) in the largest sampled KIBRA study (>2000 subjects). The interpretation was that KIBRA is not important for processes related to early memory formation, but instead relates to consolidation or delayed retention. These findings corroborate well with the present observation that KIBRA-related differences were evident in this subfield and with prior reports demonstrating high levels of expression in $\mathrm{CA}_{1}$ (Papassotiropoulos et al., 2006; Johannsen et al., 2008). Yet, other studies have observed KIBRA-related performance differences at immediate recall only (Schaper et al., 2008) or both retention intervals (Vassos et al., 2010), suggesting the effects of KIBRA on memory are nuanced.

We found no significant effects of KIBRA genotype on MTL cortex volume, suggesting that the effects of KIBRA genotype on episodic memory previously reported in the literature may be driven by differences in the hippocampus proper. However, fMRI studies have also implicated a major role of the MTL cortex in episodic memory. For example, PRC and PHC are held to encode item and context information, respectively, while the hippocampus integrates this information (Diana et al., 2007). Since contextual information is critical for recollection, $\mathrm{PHC}$ is thought to be especially important for this process. Moreover, both fMRI studies of KIBRA (Papassotiropoulos et al., 2006; Kauppi et al., 2011) observed group differences in activation that extended into the MTL cortices, although the specific localization is unclear.

To address this issue, we performed exploratory post hoc analyses, which revealed no group differences for PRC $(p=0.44$ $\left.\mathrm{hp}^{2}=.02\right)$ or ERC $\left(p=0.84, \mathrm{hp}^{2}=0.001\right)$, while PHC volume was slightly larger in T-carriers relative to noncarriers, with a marginally significant group difference $\left(F_{(1,29)}=3.77, p=0.06\right.$, $\left.\mathrm{hp}^{2}=0.12\right)$. This subtle difference in volume may either reflect direct effects of KIBRA on PHC or a downstream neuroplastic effect resulting from KIBRA's effects on the hippocampus and associated mnemonic advantage in T-carriers observed previously. While Papassotiropoulos et al. (2006) reported that the highest levels of KIBRA expression were in the hippocampus and the temporal lobes (encompassing the entire temporal lobe and hippocampus) in humans, the specific localization of expression within the temporal lobes was not examined.

An important caveat is that our protocol does not differentiate between DG and $\mathrm{CA}_{2 / 3}$. Hence, it is possible that we diluted specific effects of one of these subregions. Akin to previous investigations (Zeineh et al., 2000; Mueller et al., 2007; Olsen et al., 2009; Das et al., 2012) we used relatively thicker slices resulting in anisotropic voxels, motivated, in part, by the hippocampal atlases used for landmark demarcation that employ $\sim 2-4 \mathrm{~mm}$ thick slices (Amaral and Insausti, 1990; Duvernoy, 2005). Moreover, the layered structure of the hippocampus is best appreciated coronally, where the resolution was high. Nonetheless, given the resolution, our segmentation scheme was limited to the hippocampal body and most posterior head slices. Accordingly, future studies using higher resolution to segment across the entire hippocampal long axis are needed to assess the specificity of the KIBRA-subregion association reported here and confirm these preliminary findings. Complementary data from human histological studies of $K I B R A$ are also required. 
Given differences in MTL segmentation reported in the literature, methodological factors cannot be ruled out as contributing to the results. Indeed, we observed KIBRA effects on whole hippocampus, while Papassotiropoulos et al. (2006) failed to observe effects at a similar resolution. Likewise, the subregion-specific findings reported here may be due to the segmentation scheme adopted and, therefore, may not be observed with a different labeling approach used by other researchers. Additionally, the null effect of KIBRA genotype on subiculum volume in the present study may have been related to the difficulty in demarcating this structure, where reliability was low relative to the other regions, a finding reported previously (Mueller et al., 2007; Bonnici et al., 2012). Finally, while subfield protocols rely heavily on the use of atlases to determine the placement of landmarks, for reliability, arbitrary landmarks are also sometimes used as a "best guess" in situations where certain borders are less clear, representing a trade-off between validity and reliability. This represents a challenge in this field that may be aided by future studies mapping subregion segmentation from postmortem (ex vivo) MRI onto that of in vivo imaging.

Another limitation is that our sample was predominantly female. Previous research has shown that sex modifies the relationship between KIBRA and cognition with larger genotype effects in females (Wersching et al., 2011). Given the small number of males, we were not able to address this issue, although covarying sex in the analysis did not alter the pattern of results.

KIBRA-related differences were observed in the absence of behavioral differences, as groups were deliberately performance matched. Likewise, two previous fMRI studies have reported KIBRA-related differences in hippocampal and MTL cortex BOLD response, in the absence of performance differences $(\mathrm{Pa}-$ passotiropoulos et al., 2006; Kauppi et al., 2011; due to matching). The purpose of performance matching is to ensure that neural differences are not driven by behavioral effects per se (Rasch et al., 2010). As noted by Kauppi et al. (2011), this approach does not necessarily eliminate all behavioral differences; accordingly, the genotype-dependent differences in brain structure or $B O L D$ response may reflect qualitative differences in the integrity of the memory trace (e.g., deeper encoding, greater vividness), which are not necessarily captured by the particular task used for performance matching.

Identifying a putative neural mechanism for KIBRA's effects on human memory may have clinical utility. Recent research suggests that the hippocampal subfields are differentially associated with various neurological conditions. For example, $\mathrm{CA}_{1}$ is an early target site of pathology in Alzheimer's disease (AD; Kerchner et al., 2012). Moreover, a recent meta-analysis also suggests an association between KIBRA and risk of AD (Burgess et al., 2011) although the findings have been somewhat mixed with respect to the direction of the allelic-specific effect (Hayashi et al., 2010). Future studies are needed to explore further the complex relationship between KIBRA, cognition, and disease-related neuropathology within the hippocampus. It would also be useful to examine hippocampal volume longitudinally, to determine whether the effects of KIBRA accumulate with age.

\section{References}

Amaral DG, Insausti R (1990) Hippocampal formation. In: The human nervous system (Paxinos G, ed), pp 711-755. San Diego: Academic.

Arndt S, Cohen G, Alliger RJ, Swayze VW 2nd, Andreasen NC (1991) Problems with ratio and proportion measures of imaged cerebral structures. Psychiatry Res 40:79-89. CrossRef Medline

Bates TC, Price JF, Harris SE, Marioni RE, Fowkes FG, Stewart MC (2009)
Association of KIBRA and memory. Neurosci Lett 458:140-143. CrossRef Medline

Bonnici HM, Chadwick MJ, Kumaran D, Hassabis D, Weiskopf N, Maguire EA (2012) Multi-voxel pattern analysis in human hippocampal subfields. Front Hum Neurosci 6:290. Medline

Burgess JD, Pedraza O, Graff-Radford NR, Hirpa M, Zou F, Miles R, Nguyen T, Li M, Lucas JA, Ivnik RJ, Crook J, Pankratz VS, Dickson DW, Petersen RC, Younkin SG, Ertekin-Taner N (2011) Association of common KIBRA variants with episodic memory and AD risk. Neurobiol Aging 32:557.e1-557.e9. CrossRef Medline

Carr VA, Rissman J, Wagner AD (2010) Imaging the human medial temporal lobe with high-resolution fMRI. Neuron 65:298-308. CrossRef Medline

Chen J, Olsen RK, Preston AR, Glover GH, Wagner AD (2011) Associative retrieval processes in the human medial temporal lobe: hippocampal retrieval success and CA1 mismatch detection. Learn Mem 18:523528. CrossRef Medline

Collins DL, Holmes CJ, Peters TM, Evans AC (1995) Automatic 3-D modelbased neuroanatomical segmentation. Hum Brain Mapp 3:190-208. CrossRef

Das SR, Avants BB, Pluta J, Wang H, Suh JW, Weiner MW, Mueller SG, Yushkevich PA (2012) Measuring longitudinal change in the hippocampal formation from in vivo high-resolution T2-weighted MRI. Neuroimage 60:1266-1279. CrossRef Medline

Diana RA, Yonelinas AP, Ranganath C (2007) Imaging recollection and familiarity in the medial temporal lobe: a three-component model. Trends Cogn Sci 11:379-386. CrossRef Medline

Dice L (1945) Measures of the amount of ecologic association between species. Ecology 26.

Duvernoy H (2005) Functional anatomy, vascularization and serial sections with MRI. In: The human hippocampus, Ed 3, p 232. Berlin: Springer.

Eichenbaum H, Yonelinas AP, Ranganath C (2007) The medial temporal lobe and recognition memory. Annu Rev Neurosci 30:123-152. CrossRef Medline

Hayashi N, Kazui H, Kamino K, Tokunaga H, Takaya M, Yokokoji M, Kimura R, Kito Y, Wada T, Nomura K, Sugiyama H, Yamamoto D, Yoshida T, Currais A, Soriano S, Hamasaki T, Yamamoto M, Yasuda Y, Hashimoto R, Tanimukai H, et al. (2010) KIBRA genetic polymorphism influences episodic memory in Alzheimer's disease, but does not show association with disease in a Japanese cohort. Dement Geriatr Cogn Disord 30:302-308. CrossRef Medline

Insausti $\mathrm{R}$, Juottonen $\mathrm{K}$, Soininen $\mathrm{H}$, Insausti AM, Partanen $\mathrm{K}$, Vainio $\mathrm{P}$, Laakso MP, Pitkänen A (1998) MR volumetric analysis of the human entorhinal, perirhinal, and temporopolar cortices. AJNR 19:659-671. Medline

Johannsen S, Duning K, Pavenstädt H, Kremerskothen J, Boeckers TM (2008) Temporal-spatial expression and novel biochemical properties of the memory-related protein KIBRA. Neuroscience 155:1165-1173. CrossRef Medline

Kauppi K, Nilsson LG, Adolfsson R, Eriksson E, Nyberg L (2011) KIBRA polymorphism is related to enhanced memory and elevated hippocampal processing. J Neurosci 31:14218-14222. CrossRef Medline

Kerchner GA, Deutsch GK, Zeineh M, Dougherty RF, Saranathan M, Rutt BK (2012) Hippocampal CA1 apical neuropil atrophy and memory performance in Alzheimer's disease. Neuroimage 63:194-202. CrossRef Medline

Milnik A, Heck A, Vogler C, Heinze HJ, de Quervain DJ, Papassotiropoulos A (2012) Association of KIBRA with episodic and working memory: a meta-analysis. Am J Med Genet B Neuropsychiatr Genet 159B:958-969. CrossRef Medline

Mueller SG, Stables L, Du AT, Schuff N, Truran D, Cashdollar N, Weiner MW (2007) Measurement of hippocampal subfields and age-related changes with high resolution MRI at 4T. Neurobiol Aging 28:719-726. CrossRef Medline

Mueller SG, Chao LL, Berman B, Weiner MW (2011) Evidence for functional specialization of hippocampal subfields detected by MR subfield volumetry on high resolution images at $4 \mathrm{~T}$. Neuroimage 56:851-857. CrossRef Medline

Mueller SG, Laxer KD, Scanlon C, Garcia P, McMullen WJ, Loring DW, Meador KJ, Weiner MW (2012) Different structural correlates for verbal memory impairment in temporal lobe epilepsy with and without me- 
sial temporal lobe sclerosis. Hum Brain Mapp 33:489-499. CrossRef Medline

Olsen RK, Nichols EA, Chen J, Hunt JF, Glover GH, Gabrieli JD, Wagner AD (2009) Performance-related sustained and anticipatory activity in human medial temporal lobe during delayed match-to-sample. J Neurosci 29:11880-11890. CrossRef Medline

Olsen RK, Palombo DJ, Rabin JS, Levine B, Ryan JD, Rosenbaum RS (2013) Volumetric analysis of medial temporal lobe subregions in developmental amnesia using high-resolution magnetic resonance imaging. Hippocampus. Advance online publication. Retrieval date July 5, 2013. doi: 10.1002/ hipo.22153. CrossRef Medline

O'Reilly RC, Rudy JW (2001) Conjunctive representations in learning and memory: principles of cortical and hippocampal function. Psychol Rev 108:311-345. CrossRef Medline

Papassotiropoulos A, de Quervain DJ (2011) Genetics of human episodic memory: dealing with complexity. Trends Cogn Sci 15:381-387. CrossRef Medline

Papassotiropoulos A, Stephan DA, Huentelman MJ, Hoerndli FJ, Craig DW, Pearson JV, Huynh KD, Brunner F, Corneveaux J, Osborne D, Wollmer MA, Aerni A, Coluccia D, Hänggi J, Mondadori CR, Buchmann A, Reiman EM, Caselli RJ, Henke K, de Quervain DJ (2006) Common Kibra alleles are associated with human memory performance. Science 314: 475-478. CrossRef Medline

Rasch B, Papassotiropoulos A, de Quervain DF (2010) Imaging genetics of cognitive functions: focus on episodic memory. Neuroimage 53: 870-877. CrossRef Medline

Rolls ET, Kesner RP (2006) A computational theory of hippocampal function, and empirical tests of the theory. Prog Neurobiol 79:1-48. CrossRef Medline
Rudebeck SR, Scholz J, Millington R, Rohenkohl G, Johansen-Berg H, Lee AC (2009) Fornix microstructure correlates with recollection but not familiarity memory. J Neurosci 29:14987-14992. CrossRef Medline

Schaper K, Kolsch H, Popp J, Wagner M, Jessen F (2008) KIBRA gene variants are associated with episodic memory in healthy elderly. Neurobiol Aging 29:1123-1125. CrossRef Medline

Tulving E (2002) Episodic memory: from mind to brain. Annu Rev Psychol 53:1-25. CrossRef Medline

Vassos E, Bramon E, Picchioni M, Walshe M, Filbey FM, Kravariti E, McDonald C, Murray RM, Collier DA, Toulopoulou T (2010) Evidence of association of KIBRA genotype with episodic memory in families of psychotic patients and controls. J Psychiatr Res 44:795-798. CrossRef Medline

Wersching H, Guske K, Hasenkamp S, Hagedorn C, Schiwek S, Jansen S, Witte V, Wellmann J, Lohmann H, Duning K, Kremerskothen J, Knecht S, Brand E, Floel A (2011) Impact of common KIBRA allele on human cognitive functions. Neuropsychopharmacology 36:1296-1304. CrossRef Medline

Yassa MA, Lacy JW, Stark SM, Albert MS, Gallagher M, Stark CE (2011) Pattern separation deficits associated with increased hippocampal CA3 and dentate gyrus activity in nondemented older adults. Hippocampus 21:968-979. Medline

Yoshihama Y, Hirai T, Ohtsuka T, Chida K (2009) KIBRA Colocalizes with protein kinase Mzeta (PKMzeta) in the mouse hippocampus. Biosci Biotechnol Biochem 73:147-151. CrossRef Medline

Zeineh MM, Engel SA, Bookheimer SY (2000) Application of cortical unfolding techniques to functional MRI of the human hippocampal region. Neuroimage 11:668-683. CrossRef Medline 